IOS Press

\title{
Inferring loneliness levels in older adults from smartphones
}

\author{
Wendy Sanchez ${ }^{\text {a, }}$, Alicia Martinez ${ }^{\mathrm{a}}$, Wilfrido Campos ${ }^{\mathrm{a}}$, Hugo Estrada ${ }^{\mathrm{b}}$ and Vicente Pelechano ${ }^{\mathrm{c}}$ \\ a Department of Computer Science CENIDET, Interior Internado Palmira S/N, Palmira, Cuernavaca, México \\ E-mail: \{wendy.sanchez11c,amartinez,mcwilfrido11c\}@cenidet.edu.mx \\ ${ }^{\mathrm{b}}$ INFOTEC, San Fernando 37, Toriello Guerra, Distrito Federal, Mexico \\ E-mail: hugo.estrada@infotec.com.mx \\ ${ }^{\mathrm{c}}$ Centro de Investigación en Métodos de Producción de Software, Univesitat Politécnica de València, Camino de \\ Vera s/n, 46022 Valencia, Spain \\ E-mail:pele@pros.upv.es
}

\begin{abstract}
The number of older adult has increased significantly in most current societies. One problem that is accentuated in the stage of old age is loneliness which is a serious health risk. Therefore, new methods for early detection of this condition that make use of new non-intrusive technologies are required. Loneliness includes four main factors (family, spousal, social and existential crisis). In this paper, four predictive models to determine the level of loneliness of each factor are proposed, focusing on the activities that can be monitored using a Smartphone. Predictive models have been evaluated on basis of their accuracy, sensitivity, specificity, predictive values, type I and type II error rates. This paper also presents the results of the experimentation of the proposed approach in practice and with real users through a mobile application called " $i$ Vive!" that implements the predictive models.
\end{abstract}

Keywords: Loneliness, predictive models, older adults, ESTE-R scale

\section{Introduction}

Nowadays, in many countries worldwide, the number of older adults is increasing. In Mexico, for instance, the population is aging due a greater life expectancy, combined with a significant decrease in the fertility rate [1]. In 2030, older adults will represent 14.8 percent of the population [2].

One of the social problems that older adults face is loneliness. Possible causes include retirement and the death of a spouse and/or friends, among others. Loneliness is a multidimensional psychological condition comprised of cognitive, emotional, behavioral and social variables [3]. It is divided into two types: objective loneliness and subjective loneliness. The first refers to a lack of company. This does not always imply an unpleasant experience. It may even become a desirable and enjoyable experience [4]. The second type includes a painful emotional condition. It includes situ-

* Corresponding author. ations of vulnerability, marginalization, and exclusion which result in the person feeling misunderstood, rejected, and also needing company in order to perform physical and intellectual activities [5]. Loneliness occurs when social relationships of a person are fewer and less satisfactory than that which is desired [6]. Loneliness can even be experienced when one has contact with other people [7]. Also, loneliness is considered to be one of the possible factors that causes disorders such as depression, suicide, and serious medical problems like heart illnesses [8]. This situation has sparked an interest and concern to improve the wellbeing and quality of life for this segment of the population.

Currently, research in the diagnostic field of loneliness amongst the elderly is developing, particularly in the analysis of human behavior that gives rise to this disorder. From the psychological point of view, many self-report based instruments have been developed to assess loneliness in older adults. These instruments are questionnaires made up of a series of questions re- 
lated to factors that give rise to this emotional condition. Rubio Herrera [9] proposes the ESTE-R scale, an instrument that consists of 34 items that consider different aspects of the concept of loneliness. It focuses on four factors: family loneliness, spousal loneliness, social loneliness and existential crisis loneliness. For this last factor, Russell, D.W. [10] proposes the UCLA loneliness scale which also incorporates questions about feelings of social isolation. Unfortunately, these instruments require an application routine which could be bothersome.

Determining loneliness in a timely manner is fundamental in offering medical and technological treatment for this disorder. However, this must be done in the least intrusive way possible in order to facilitate its utilization in practice. This calls for the use of innovative technology that permits using everyday devices to monitor activities associated to loneliness.

In this sense, Ambient Intelligence is looking to create a digital environment that would help these people in their daily lives in a manner that is simple, that is to say, intelligent [11], ubiquitous and proactive at the same time [12].

Our research focuses on automatically inferring the older adults' loneliness level through activities that can be monitored by a Smartphone. In this paper, attributes that have a correlation with the factors of loneliness are identified [10]. Using such attributes, four predictive models to infer each factor of loneliness were developed, through investigation of a combination of key aspects, including a range of classification algorithms, relevant attributes subsets, and data imbalance handling. Next, the results of an experiment through implementation of all models in a mobile application are presented.

Such models could become a part of a screening process, in order to carry out suitable interventions that allow older adults to cope with this health condition.

The paper is structured as follows: Section 2 describes the related works that detect loneliness. In Section 3 the proposed predictive models of Loneliness are presented. In Section 4 a mobile application called "iVive!" is given. Section 5 shows a performance experimental test. Section 6 discusses the results; and finally, Section 7 presents the conclusions and future work.

\section{Related works}

Computer science has contributed to the diagnosis of loneliness. Shigeki Aoki et al. [13] identify loneli- ness in older adults from inside their homes by placing infrared sensors in various areas (the living room, the dining room, the bathroom, etc.). The data retrieved from the sensors was then shown as a sequence of tags and afterward analyzed with the Hidden Markov model. Besides, Yeong Hyeon Gu et al. [14] considered the use of RFID tags located in furniture and household appliances. A reader glove attached to the hand of the older adult collected readings corresponding to the object being used and the time of use. This criteria was used to determine if the elderly person was experiencing loneliness. This process also took into consideration the time during which the person was not engaged in any activity and when he or she was performing an activity for an extended period of time. However, these proposals required the installation of special equipment in the home, and this equipment only collected data when the person was at home. Our approach proposes inferring loneliness through a Smartphone, something that is already part of daily life, without the need for the installation and configuration of sensors in the home of the elderly person.

In [15] it is suggested that a large variability in outof-home profiles may allow detection of individuals at higher risk of loneliness. On the other hand, [16] focuses on analysis of loneliness through mobile phones and the results revealed that loneliness was significantly associated with problematic mobile phone use. Our proposed approach used attributes that have been demonstrated to have a correlation with loneliness recognition $[15,16]$.

\section{Predictive models}

In data mining, data scientists use algorithms to identify previously unrecognized patterns and trends hidden within vast amounts of structured and unstructured information. These patterns are used to create predictive models that try to forecast future behavior. A predictive model examines an attribute set and produces an outcome class. Our research work focuses on identifying attributes that have a correlation with loneliness. These attributes correspond to activities performed by older adults that can be monitored by Smartphones, such as: cellular phone use, the number of outings taken from home, the number of activities performed at home and the time spent inside/outside of the house $[15,16]$.

Using such attributes, four predictive models were developed to infer each factor of loneliness (family, 
spousal, social and existential crisis). In order to develop the most suitable models, a range of popular data mining algorithms were evaluated. Additionally, due to an imbalance of class sizes in the collected data, a resampling technique for building the prediction models was used. Each individual data mining algorithm was then applied to data with different attribute sets, on both scenarios with and without data resampling to handle the data imbalance. This was carried out through WEKA [17], which is an experimentation space for data analysis. It contains a collection of machine learning algorithms that permit testing, analyzing and evaluating data mining tasks such as preprocessing, classification, grouping, association, selection of attributes and visualization.

It is important to point out that the predictive models represent a relevant contribution of the research work. It has been developed following a systematic process using real data of elderly people in order to ensure the precision of the models. Following, these phases are described in detail.

\subsection{Data collection}

The data collection was done through a questionnaire applied to 100 older adults, including both men and women between 60 and 90 years of age $(69 \pm$ 7.165), with full mental and physical capacities, who also use a cellular phone, had no difficulty understanding the questions, signed the inform consent and were willing to take part of the research. Older adults with physical, sensory or cognitive disability were excluded from the study, in order to assure that the results of the experiment would not be affected by the limitations of the participants. The questionnaires were given in two different cities in places where older adults congregated such as: churches, dance centers, retirement homes, hospitals and esthetic salons.

The questionnaire comprises two parts. The first one gathered the level of loneliness in the older adult through the ESTE-R scale [9] which is targeted to Spanish speakers. This scale includes different dimensions of loneliness and groups them into four factors: family loneliness, spousal loneliness, social loneliness and existential crisis. The scale is comprised of 34 items and the response options are: never, rarely, sometimes, regularly, or always. The Cronbach's Alpha is of 0.915, which indicates an excellent internal consistency. The scale results reflect the subject's loneliness level and are valid for one month before the test is ad-
Table 1

Number of older adults in each factor of loneliness with the ESTE-R scale

\begin{tabular}{lccr}
\hline Factor of loneliness & Low & Medium & High \\
\hline Family loneliness & 81 & 17 & 2 \\
Spousal loneliness & 46 & 7 & 47 \\
Social loneliness & 71 & 23 & 6 \\
Existential crisis & 70 & 24 & 6 \\
\hline
\end{tabular}

Table 2

Demographic information about the older adults

\begin{tabular}{llc}
\hline Variable & Categories & Percentage \\
\hline Sex & Female & $54 \%$ \\
& Male & $46 \%$ \\
Civil Status & With Partner & $55 \%$ \\
& Single & $45 \%$ \\
Suffering from Illness & Yes & $66 \%$ \\
& No & $34 \%$ \\
Working Situation & Employed & $22 \%$ \\
& Unemployed & $78 \%$ \\
\hline
\end{tabular}

ministered. Table 1 shows the loneliness level scores obtained by older adults in each factor of loneliness.

The second part of the questionnaire collected the activities performed by the older adults during the last week before the questionnaire was applied. The questions refer to four activity categories: cellular phone use, number of outings from home, number of activities performed at home and time spent inside/outside of home. In order to help older adults remember their activities various detailed questions were asked and later were joined together. The number of outings is the sum of the number of outings to the supermarket, restaurants, church, to visit their family, to visit their friends and doctors. The average time at home is the sum of the average time in the bedroom, living room and kitchen. In addition, participants were asked demographic questions asking for their age, sex, civil status, health conditions and working situation. The data collected by all the questions is shown in Tables 2 5 and 6 . The data was compiled into four files, one for each factor of loneliness. Every file contains 14 attributes about the activities plus the output class: the level of loneliness.

\subsection{Attribute selection}

Attribute selection is the process of identifying and removing as much irrelevant and redundant informa- 
Table 3

Cellular phone use of the older adult

\begin{tabular}{|c|c|c|c|}
\hline Attribute & Minimum value & Maximum value & Average \\
\hline Outgoing calls to family & 0 calls & 30 calls & $4.25 \pm 6.12$ calls \\
\hline Outgoing calls to friends & 0 calls & 35 calls & $2.53 \pm 5.24$ calls \\
\hline Outgoing calls to acquaintances & 0 calls & 35 calls & $1.62 \pm 4.54$ calls \\
\hline Incoming calls from family & 0 calls & 30 calls & $4.52 \pm 6.17$ calls \\
\hline Incoming calls from friends & 0 calls & 40 calls & $2.38 \pm 5.33$ calls \\
\hline Incoming calls from acquaintances & 0 calls & 21 calls & $1.22 \pm 2.95$ calls \\
\hline Outgoing messages to family & 0 messages & 50 messages & $1.74 \pm 5.98$ messages \\
\hline Outgoing messages to friends & 0 messages & 25 messages & $0.7 \pm 3.04$ messages \\
\hline Outgoing messages to acquaintances & 0 messages & 10 messages & $0.32 \pm 1.35$ messages \\
\hline Incoming messages from family & 0 messages & 50 messages & $1.54 \pm 5.63$ messages \\
\hline Incoming messages from friends & 0 messages & 25 messages & $0.95 \pm 3.26$ messages \\
\hline Incoming messages from acquaintances & 0 messages & 20 messages & $0.52 \pm 2.26$ messages \\
\hline
\end{tabular}

Table 4

Frequency of number of outings from the home of the older adult (Absolute frequency - Relative frequency)

\begin{tabular}{lccc}
\hline Attribute & 0 outgoings & From 1 to 2 outgoings & 3 or more outgoings \\
\hline Number of trips to the supermarket & $24-24 \%$ & $44-44 \%$ & $32-32 \%$ \\
Number of visits to shopping centers & $49-49 \%$ & $38-38 \%$ & $38-38 \%$ \\
Number of trips to restaurants & $33-33 \%$ & $40-40 \%$ & $29-29 \%$ \\
Number of trips to church & $40-40 \%$ & $39-39 \%$ & $20-20 \%$ \\
Number of visits with family & $49-49 \%$ & $38-38 \%$ & $12-12 \%$ \\
Number of visits with friends & $57-57 \%$ & $33-33 \%$ & $5-5 \%$ \\
Number of doctor appointments & $63-63 \%$ & $4-4 \%$ \\
\hline
\end{tabular}

Table 5

Frequency of activities performed in the home of the older adult (Absolute frequency - Relative frequency)

\begin{tabular}{lccc}
\hline Attribute & 0 times & From 1 to 2 times & 3 or more times \\
\hline Cleaning the house & $35-35 \%$ & $29-29 \%$ & $36-36 \%$ \\
Laundry & $39-39 \%$ & $45-45 \%$ & $16-16 \%$ \\
Gardening & $50-50 \%$ & $31-31 \%$ & $19-19 \%$ \\
Other activities & $46-46 \%$ & $23-23 \%$ & $31-31 \%$ \\
\hline
\end{tabular}

Table 6

Time spent inside and outside of the home of the older adult

\begin{tabular}{|c|c|c|c|c|c|}
\hline Attribute & 0 hours & 1 to 3 hours & 4 to 7 hours & 8 to 10 hours & More than 10 hours \\
\hline Average time in the bedroom (excluding sleep time) & $25-25 \%$ & $43-43 \%$ & $13-13 \%$ & $9-9 \%$ & $10-10 \%$ \\
\hline Average time in the living room & $18-18 \%$ & $36-36 \%$ & $26-26 \%$ & $11-11 \%$ & $9-9 \%$ \\
\hline Average time in the kitchen & $27-27 \%$ & $32-32 \%$ & $19-19 \%$ & $13-13 \%$ & $9-9 \%$ \\
\hline Average time spent outside of the house & $16-16 \%$ & $12-12 \%$ & $19-19 \%$ & $16-16 \%$ & $37-37 \%$ \\
\hline
\end{tabular}

tion as possible. As a result there was an improvement in the process's predictive performance, a decrease in its elapsed time, and a reduction in its storage needs. At the same time, a better view and understanding of the data was obtained. Most machine learning algorithms are designed to learn which are the most appropriate attributes to use for making their decisions. Decision tree methods choose the most promising attribute to split at each point and should in theory never select irrelevant or unhelpful attributes [18]. In order to obtain the first subset of relevant attributes, J48 classification algorithm was applied to the full 
Table 7

Relevant attributes subset obtained

\begin{tabular}{|c|c|}
\hline Factor & Relevant attributes subset \\
\hline Family Loneliness & $\begin{aligned} \mathrm{V} 1= & \{\text { Outgoing calls to family, Average time } \\
& \text { outside of the home }\}\end{aligned}$ \\
\hline Spousal Loneliness & $\begin{aligned} \mathrm{V} 2= & \{\text { Civil Status, Outgoing calls to family, } \\
& \text { Incoming calls from family, Total } \\
& \text { outings }\}\end{aligned}$ \\
\hline Social Loneliness & $\begin{aligned} \mathrm{V} 3= & \text { \{ Outgoing calls to acquaintances, Total } \\
& \text { outings, Average time outside of the } \\
& \text { home }\}\end{aligned}$ \\
\hline Existential Crisis & $\begin{aligned} \mathrm{V} 4= & \{\text { Sex, Civil Status, Illness, Working } \\
& \text { Situation, Average time outside of the } \\
& \text { home }\}\end{aligned}$ \\
\hline
\end{tabular}

dataset. Then, the subset obtained was assessed using Chi-Squared [19] and Ranker methods for evaluation of attributes, Correlation-based Feature Selection and Greedy Stepwise [20] for evaluation of the sets of attributes and Greedy Stepwise method and J48 classification algorithm for the evaluation of the Wrapper attribute sets [21]. All the tests were performed with a 10-fold cross validation as the standard evaluation technique [18]. Table 7 shows the resulting relevant attributes subset for each factor of loneliness.

\subsection{Classification}

In order to develop the most suitable models for the loneliness prediction, a range of classifier algorithms were assessed [22], one of each category available in WEKA [17]. ZeroR (ZR) algorithm was used as baseline. The other classifier algorithms used were NaiveBayes (NB) [23] from Bayes algorithms, Simple Logistic (SL) [24] and Support Vector Machine (SVM) [25] from function algorithms, k-NearestNeighbor (kNN) [26] from lazy algorithms, AdaBoost (AB) [27] from meta algorithms, OneR (OR) [28] from rules algorithms and J48 [29] and SimpleCart (SC) [30]s from tree-based algorithms. A stratified ten times ten-fold cross-validation technique was used because it is the standard evaluation technique in situations where only limited data is available [18].

\subsection{Balancing the dataset}

A dataset is imbalanced if the classification categories are not equally represented [31]. The imbalance between such class data can have an impact on some classification algorithms, typically with bias toward the majority class prediction. Therefore, applying a dataset balancing technique is required.
Table 8

Number of older adults in each factor of loneliness obtained with SMOTE

\begin{tabular}{lccc}
\hline Factor of loneliness & Low & Medium & High \\
\hline Family loneliness & 81 & 85 & 82 \\
Spousal loneliness & 46 & 42 & 47 \\
Social loneliness & 71 & 69 & 66 \\
Existential crisis & 70 & 72 & 66 \\
\hline
\end{tabular}

In almost all of the factors of loneliness, there is strong bias towards the "Low" class. In order to handle the imbalance, the dataset was resampled by applying the synthetic minority oversampling technique (SMOTE) [31]. Each derived model is denoted by the name of the classifier algorithm plus "_S" when the SMOTE is applied. For example, a model derived using $\mathrm{kNN}$ classification and SMOTE for data resampling is denoted as "kNN_S" and without data resampling is denoted as "kNN". Table 8 shows the dataset balanced after applying SMOTE.

\subsection{Models evaluation}

Predictive models performance was evaluated in terms of accuracy [32], sensitivity, specificity, positive and negative predictive values [33] and error types I and II. In order to corroborate the results of the predictive models, a reference standard was necessary to define an alternative and real diagnosis. The reference standard used was the ESTE-R scale.

The predictive model results compared to the real reference standard results could be grouped in four types:

- True positive $(T P)$. The model predicts a presence of loneliness in the older adult when in fact it is a presence.

- True negative $(T N)$. The model predicts an absence of loneliness in the older adult when in fact it is an absence.

- False Positive $(F P)$. The model predicts a presence of loneliness in the older adult when in fact it is an absence.

- False negative $(F N)$ The model predicts an absence of loneliness in the older adult when in fact it is a presence.

Table 9 shows these results, which conform the confusion matrix, a $2 \times 2$ table. The rows correspond to the predictive model results and the columns correspond to the ESTE-R scale results. 
Table 9

Confusion matrix

\begin{tabular}{llll}
\hline & & \multicolumn{2}{c}{ ESTE-R Scale } \\
& & \multicolumn{1}{c}{ Positive } & \multicolumn{1}{c}{ Negative } \\
\hline & Positive & True positive & False positive \\
Predictive Model & & $(\mathrm{TP})$ & $(\mathrm{TP})$ \\
& Negative & False negative & True negative \\
& & $(\mathrm{FN})$ & $(\mathrm{FN})$ \\
\hline
\end{tabular}

\subsubsection{Accuracy}

The accuracy is the proportion of older adults for whom both the predictive model and the reference standard give the same result. Predictive accuracy is defined in Eq. (1).

$$
\text { Accuracy }=\frac{T P+T N}{T P+F P+T N+F N}
$$

\subsubsection{Sensitivity and specificity}

Sensitivity, also known as recall or $T P$ Rate, measures the proportion of the older adults with a loneliness condition as defined by the reference standard that are correctly identified by the predictive model. In other words, it measures how sensitive the predictive model is in detecting loneliness condition. Specificity, also known as recall or TN Rate, measures the proportion of the older adults who are free of the loneliness condition as defined by the reference standard that are correctly identified as free of the loneliness condition by the predictive model. Notice that sensitivity and specificity are always defined in comparison to the reference standard. That is, the best that the predictive model can be do is produce the same results as the reference standard [34].

Sensitivity and specificity are defined in Eqs (2) and (3) respectively.

$$
\begin{aligned}
& \text { Sensitivity }=\frac{T P}{T P+F N} \\
& \text { Specificity }=\frac{T N}{T N+F P}
\end{aligned}
$$

\subsubsection{Positive and negative predictive values}

The Positive Predictive Value $(P P V)$ and the Negative Predictive Value $(N P V)$ are the proportions of positive and negative results in statistics and diagnostic tests that are true positive and true negative results, respectively. The $P P V$ and $N P V$ describe the performance of the predictive model. A high result can be interpreted as indicating the accuracy of the predictive model [35]. $P P V$ is also known as precision. $P P V$ and $N P V$ are defined in Eqs (4) and (5) respectively.

$$
\begin{aligned}
& P P V=\frac{T P}{T P+F P} \\
& N P V=\frac{T N}{T N+F N}
\end{aligned}
$$

\subsubsection{Type I and type II errors}

Overall, if the model predicts a loneliness condition in the older adults when in fact it is free of the loneliness condition (type I error or FP Rate), a paranoia effect could have arisen. If the model predicts that the older adult is free of the loneliness condition when in fact the older adult has a loneliness condition (type II error or $F N$ Rate), a worse effect could have arisen due the possibility that the older adult might have been in serious risk of depression, suicide, and other serious medical problems like heart illnesses [8]. Hence, type II error rate was decisive for selecting the predictive models.

\subsubsection{Suitable models selection}

A first model performance evaluation was carried out with ZR as the baseline. This algorithm is the simplest classification method which relies on the target and ignores all predictors. ZR classifier simply predicts the majority class. Although there is no predictability power in ZR, it is useful for determining a baseline performance as a benchmark for other classification methods [18]. All the models, obtained from the dataset with and without using the SMOTE, produced a significantly higher accuracy than the baseline.

The second model performance evaluation was carried out in terms of accuracy for each factor of loneliness. The classification algorithms were applied to the data using the relevant attribute subsets of each factor of loneliness, with and without using the SMOTE. At least, one SMOTE model produced a higher accuracy than the others models in each factor of loneliness. Except in spousal loneliness, where models without using the SMOTE produced the higher accuracy scores.

The accuracy of the baseline and the classifications algorithms applied to the dataset with and without using the SMOTE, are shown in Figs 1-4.

Once the models performance were compared based upon their accuracy, the best models in terms of sensitivity, specificity, positive and negative predictive values were examined. Nevertheless, type II error was weighted heavier than other criterion since this type of error could lead to the most adverse effect in older adults. A summary of all criteria is presented in Table 10 . 


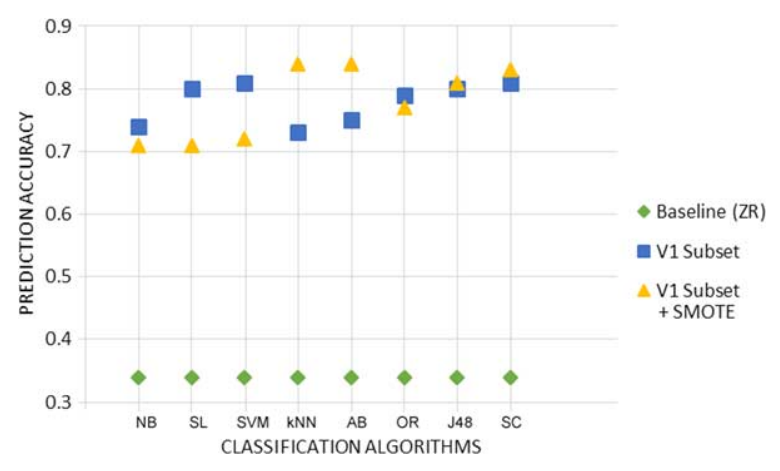

Fig. 1. Prediction accuracies for family loneliness (V1).

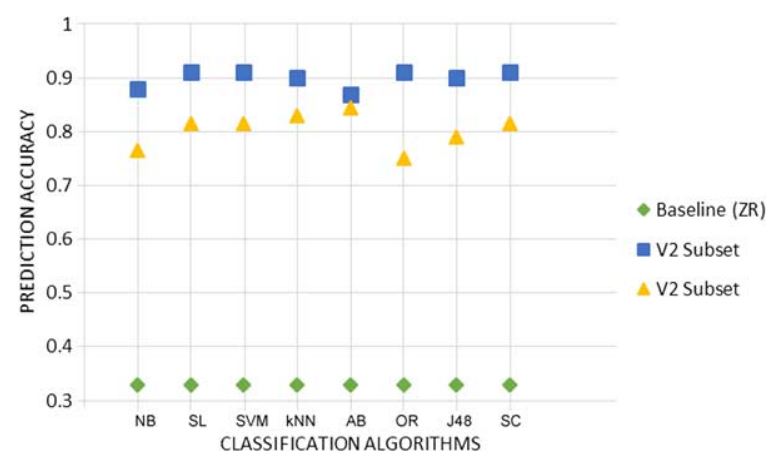

Fig. 2. Prediction accuracies for spousal loneliness (V2).

In the case of family loneliness, "kNN_S" y "AB_S" models reflected the best performance and the lowest type II error rate over the rest of models. Since both reflected the same scores, the "AB_S" model was chosen because it presented the simplest computational implementation.

In the case of spousal loneliness, "SL", "SVM", "OR", "SC" models reflected the best performance, while "kNN" reflected a slightly worse performance. Even so, "kNN" was considered the most suitable model since it reflected the lowest type II error rate.

In the case of social loneliness, "AB_S" and "J48" models reflected the same type II error rate and the same accuracy. Even so, "AB_S" was considerate the most suitable model since it reflected better scores for the rest of criterion.

In the case of existential crisis loneliness, the most suitable model was "SL_S", since it reflected the best performance and the lowest type II error rate.

\section{Mobile application: ;Vive!}

A mobile application was developed, implementing the four selected models for each factor of loneliness. This mobile application, called "¡Vive!", was capable

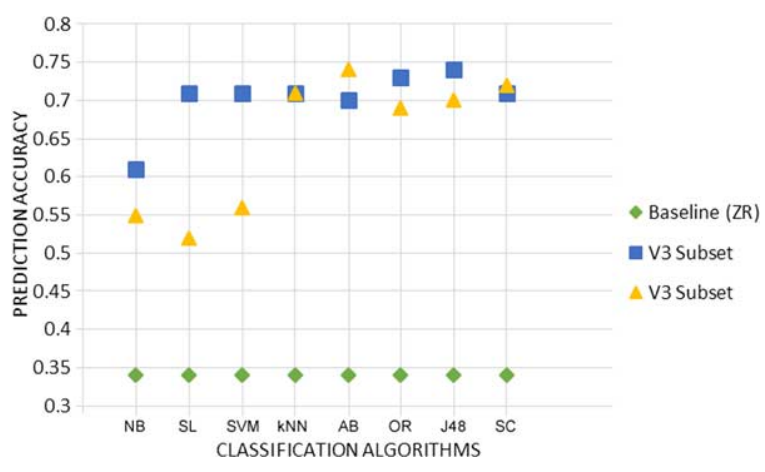

Fig. 3. Prediction accuracies for social loneliness (V3).

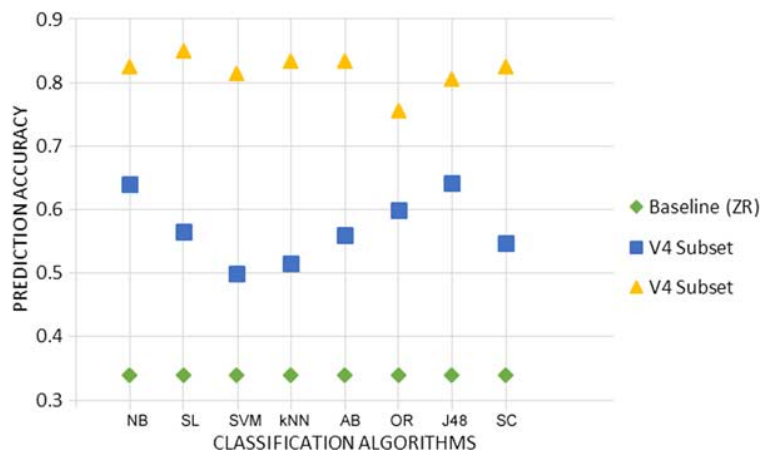

Fig. 4. Prediction accuracies for existential crisis (V4).

of monitoring the older adult's activities and determining his or her level of loneliness in relation of each factor. The mobile application " $;$ Vive!" ran on an Android operating system, between versions 2.2 Froyo (API 8) and 4.1 Jelly Bean (API 16).

The mobile application " $V$ Vive!" is comprised of four modules: the configuration module, the calls module, the monitoring module and the classifier module. Figure 5 shows the architecture of the application. Figure 6 shows a screenshot of the configuration module of the mobile application.

\subsection{Configuration module}

The configuration module requests demographic information from the older adults such as name, age, sex, civil status, health condition and working situation. The configuration module obtains the phone numbers of family members and friends with whom the older adult has the most contact through a Smartphone. This information is later used to classify the calls. Also, the configuration module obtains geographic coordinates of the older adult's home. The coordinates are obtained by communication with a GPS sensor on the Smartphone by means of the Localizer button or by writ- 
Table 10

Models prediction performance

\begin{tabular}{|c|c|c|c|c|c|c|c|c|c|}
\hline Factor of loneliness & Models & Accuracy & Sensitivity (Recall) & SPC & PPV (Precision) & PPN & F-Measure & Error type I & Error type II \\
\hline \multirow[t]{2}{*}{ Family } & “ZR" (Baseline) & 0.34 & 0.34 & 0.66 & 0.5 & 0.5 & 0.405 & 0.34 & 0.66 \\
\hline & $\begin{array}{l}\text { "kNN_S", } \\
\text { "AB_S" }\end{array}$ & 0.84 & 0.84 & 0.92 & 0.913 & 0.852 & 0.875 & 0.08 & 0.16 \\
\hline \multirow[t]{3}{*}{ Spousal } & “ZR" (Baseline) & 0.34 & 0.43 & 0.57 & 0.5 & 0.500 & 0.462 & 0.57 & 0.43 \\
\hline & $\begin{array}{l}\text { "SL", "SVM", } \\
\text { "OR", "SC" }\end{array}$ & 0.91 & 0.9 & 0.92 & 0.918 & 0.902 & 0.909 & 0.1 & 0.08 \\
\hline & "kNN" & 0.9 & 0.87 & 0.93 & 0.926 & 0.877 & 0.897 & 0.13 & 0.07 \\
\hline \multirow[t]{3}{*}{ Social } & “ZR” (Baseline) & 0.34 & 0.34 & 0.66 & 0.5 & 0.5 & 0.405 & 0.71 & 0.29 \\
\hline & "AB_S" & 0.74 & 0.74 & 0.87 & 0.851 & 0.770 & 0.791 & 0.13 & 0.26 \\
\hline & "J48" & 0.74 & 0.7 & 0.85 & 0.823 & 0.739 & 0.756 & 0.15 & 0.26 \\
\hline Existential & “ZR” (Baseline) & 0.34 & 0.35 & 0.65 & 0.5 & 0.5 & 0.412 & 0.35 & 0.65 \\
\hline Crisis & "SL_S" & 0.85 & 0.8 & 0.9 & 0.889 & 0.818 & 0.842 & 0.1 & 0.2 \\
\hline
\end{tabular}

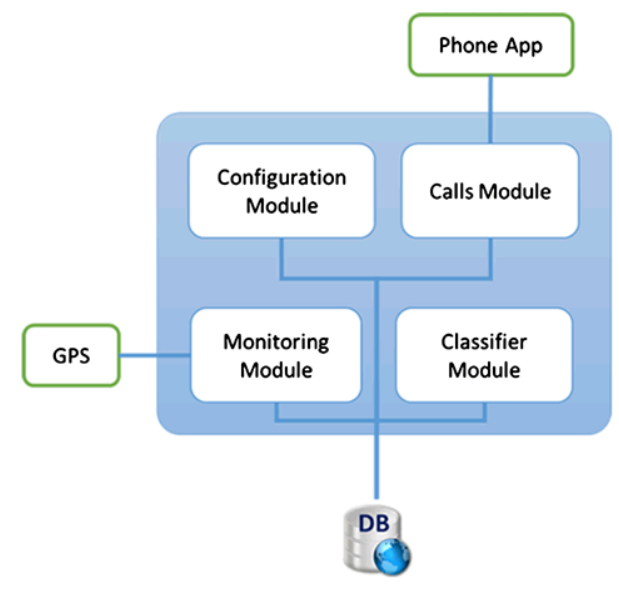

Fig. 5. Application “¡Vive!” functional modules.

ing the coordinates manually. It is necessary to obtain the house's geographic coordinates in order to be able to define the region that is considered "inside the house" and thereby determine the values of the following attributes: total number of outings and average time spent outside the home.

\subsection{Calls module}

The calls module communicates with the Smartphone application and obtains the information pertaining to calls, which was the following: type of calls (incoming or outgoing) and the telephone number in order to identify it as a family member, friend or acquaintance.

\subsection{Monitoring module}

In the monitoring module, the location of the older adult is monitored by detecting his or her geographic

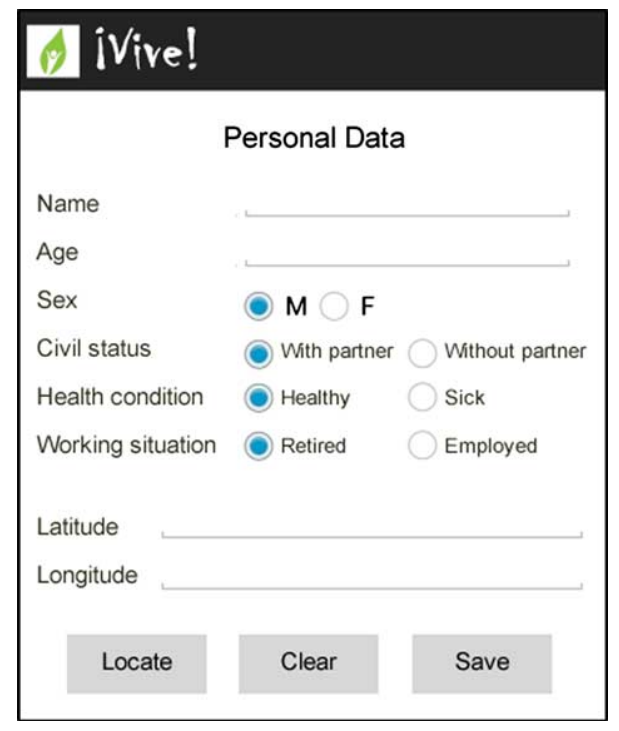

Fig. 6. Screenshot of the configuration module.

coordinates every 30 minutes. To do so the GPS sensor in the Smartphone is utilized. The monitoring occurs on a second plane in a way that is not noticeable to the user.

\subsection{Classifier module}

The classifier module gathers the registered data from one week earlier and predicts the four loneliness levels (one for each factor of loneliness) using the implemented predictive models. The information gathered refers to demographic information about the older adult, calls registered and the location of the older adults.

One of the reasons for the research is to help improve the quality of life for older adults. For this rea- 


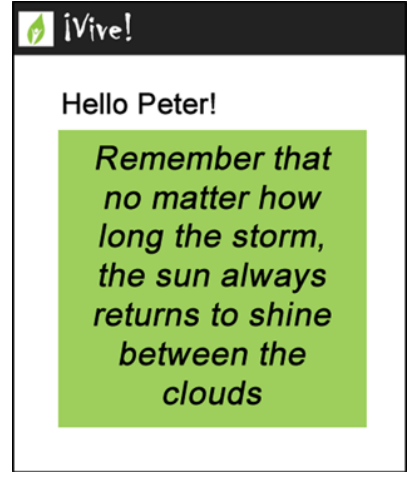

Fig. 7. Screenshot of encouragement message.

son, the level of loneliness is not revealed to the older adults as this could have been counterproductive. The results that are shown to the older adult are messages of encouragement in accordance with the level of loneliness obtained with the intention to motivate and help the older adult overcome loneliness. Figure 7 shows a screenshot with an example of an encouragement message. Table 11 shows the encouragement messages for its respective loneliness levels.

\section{Experiment}

An experiment with the mobile application " $i$ Vive!" was conducted. The purpose of the experiment was to evaluate the selected models, comparing the results of

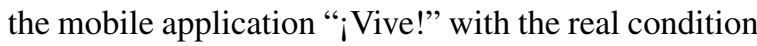
of the older adults. The self-report was not a good option since admitting the existence of a problem at the end of life may mean, for some subjects, acknowledge that he or she has failed in the family or in the society, which makes individuals really feel lonely. They may not express suffering such a problem, if you ask them directly [36]. Therefore the reference standard, the ESTE-R scale, was used.

\subsection{Materials}

The experiment included 12 older adults, three men and nine women, who met the same profile requirements as in the previous phase, with ages between 60 and 89 years old $(66.75 \pm 5.36)$. Most of them had a partner and suffer some illness like diabetes or high blood pressure. All by one of them had a job.

Seven Smartphones were used with the mobile application "Vive!" installed: 2 Samsung Galaxy Note 2 Android 4.3 mobile phones, 3 Samsung S3 An- droid 4.1.1 mobile phones and 2 LG Optimus L9 Android 4.1.2 mobile phone.

\subsection{Procedure}

The participants were asked to sign an informed consent form where they agreed to participate in the experiment. Then, the monitoring in situ of their activities was carried out through the mobile application "iVive!" over the course of a week, the participants had to carry the Smartphone to every place they went and use it in a normal manner. Every day, they were asked to charge the cellular phone and make sure it was turned on. It was not necessary for the older adults to interact with the application in any way. That is, the monitoring of the older adult's activities occurred unnoticed by the user. At the end of the week, the ESTE$\mathrm{R}$ scale was administered to each participant in order to obtain their real loneliness levels and those results were compared with the diagnostic data obtained by the mobile application " $i$ Vive!".

\subsection{Results}

Table 12 shows a summary of 12 older adults' data who were in the experimental group collected by the application of the 12 older adults in the experimental group. Column A corresponds to the number of outgoing calls to family. Column $\mathrm{B}$ corresponds to the number of outgoing calls to friends. Column $\mathrm{C}$ corresponds to the number of outgoing calls to acquaintances. Column D corresponds to the number of incoming calls from family. Column E corresponds to the number of incoming calls from friends. Column $\mathrm{F}$ corresponds to the number of incoming calls from acquaintances. Column G corresponds to the total number of outings. Column $\mathrm{H}$ corresponds to the average time spent inside the home (excluding sleep time). Column I corresponds to the average time spent outside of the home. A comparison of the loneliness levels obtained with the ESTE-R scale and with the mobile application " $\mathrm{Vive!"} \mathrm{for} \mathrm{each} \mathrm{factor} \mathrm{of} \mathrm{loneliness}$ is shown in Figs 8-11.

For family loneliness, the mobile application "¡Vive!" correctly classified 11 of 12 participants, producing an accuracy of $91.67 \%$ and a type II error rate of $0 \%$. For spousal loneliness, the mobile application " Vive!" correctly classified 10 of 12 participants, producing an accuracy of $83.34 \%$ and a type II error rate of $0 \%$. For social loneliness, the mobile application 
Table 11

Encouragement messages

Low level

"A positive thinker see an opportunity in every difficulty"

"Be proud of yourself, you are awesome"

"The best thing you can do is go out and enjoy your life"

"Be proud of the person that you are but strive to be better always"

"Today will be a wonderful day"

"Do one thing today to make you feel proud of yourself"

"Today, do something you have never done"

"Today, do something you have always wanted to do"

"Be proud of every step you take toward reaching yours goals"

"Keep improving yourself"

"Live life in the present and always be happy"

"Appreciate your life, is the most valuable gift"

Medium Level

"Face every single challenge that is coming your way"

"Love is an invaluable treasure, so you must take care and appreciate it a lot"

"Keep the positive mind to make things right"

"Have the strength to get through any barriers and achieve the goals you propose"

"Think there will be a tomorrow which will be the most radiant of all"

"Do not let your problems erase your big smile"

"The best you can do is stay calm and try to find the best solution"

"This day will be the beginning of a different life, good luck"

"Never doubt of you, trust that you are capable to make what you propose"

"If from heaven fall lemons, learn to make lemonade"

"Be very positive and help yourself to have more inner strength"

"The power of positive thinking is like car with a powerful engine that can take you to the summit of a mountain"

"Face the sun, and the shadows will remain behind you"

"There's always a tomorrow, a chance to start a new"

High level

"Appreciate life itself even if it's not a bed of roses. Contentment is not fulfillment of what you wish for but appreciation of what you have"

"There are things known, and there are things unknown. And in between are the doors"

"Life is all about a card game. Choosing the right cards is not in our hand. But playing well with the cards in hand, determines our success"

"The most determinative \& motivating sentence which should always be followed in life. The race is not over because I haven't won yet"

"Although the storm is very strong, remember that after it a beautiful rainbow that will make you remember life is wonderful will appear"

"Remember that there is always a light at the end of the tunnel. Just move on and be strong for anything or anyone makes you fall. You have the support of all the people who love you and so it will be easier to face any challenges you have"

"Use you days and all around you, enjoy the company of your loved ones, your friends and all that the life provided you"

"Promise yourself to be so strong that nothing can disturb your peace of mind"

"Believe in yourself and be proud of who you are because you are special and light your own start"

"Life is short, time is fast, no replay, no rewind. So enjoy every moment as it comes"

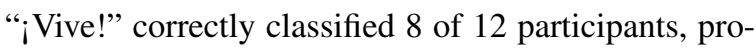
ducing an accuracy of $66.67 \%$ and a type II error rate of $0.33 \%$. For existential crisis loneliness, the mobile application “ ¡Vive!” correctly classified 10 of 12 participants, producing an accuracy of $83.34 \%$ and a type II error rate of $0 \%$.

\section{Discussion}

This research focused on inferring, in an automatic manner, the older adults' loneliness level through activities that can be monitored by a Smartphone. The attributes that have a correlation with the factors of 


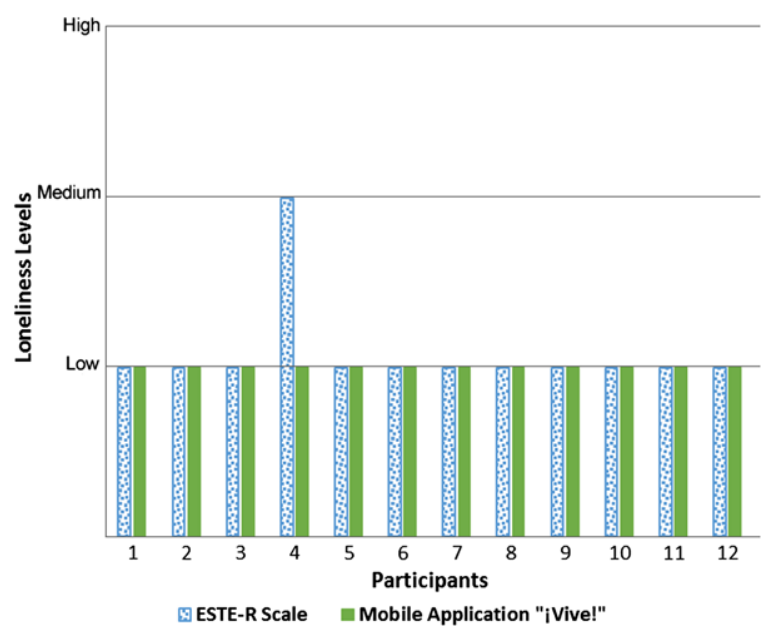

Fig. 8. Comparison results of family loneliness.

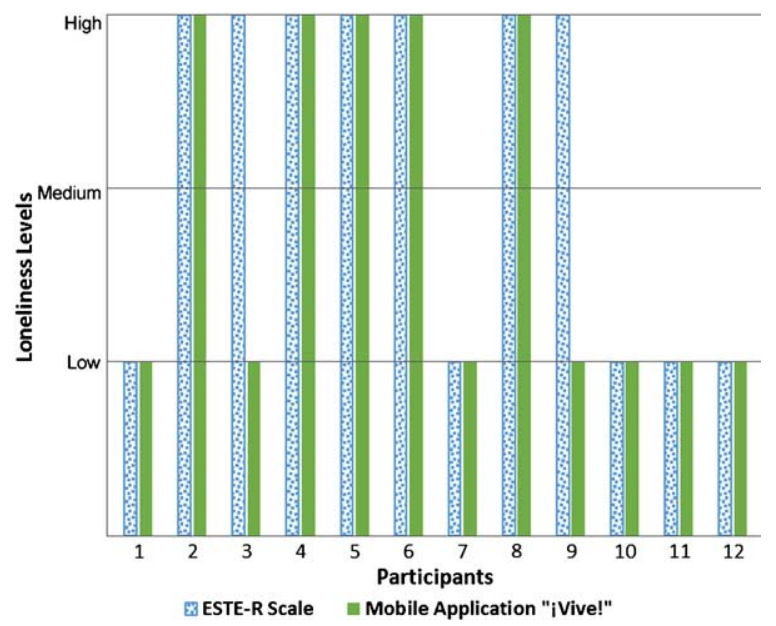

Fig. 9. Comparison results of spousal loneliness.

loneliness were identified. Also, findings on predictive models were discovered.

\subsection{Relevant attributes}

The attributes concerning of outgoing or incoming messages, did not result as a relevant attributes. One possible interpretation of this finding is that older adults do not use this type of communication because the interface is not adapted to the limitations of age.

For spousal loneliness, civil status was a discriminative attribute. One possible interpretation of this finding is that widowed older adults are strongly affected by the death of theirs spouses, so they are at a higher risk of suffering spousal loneliness.

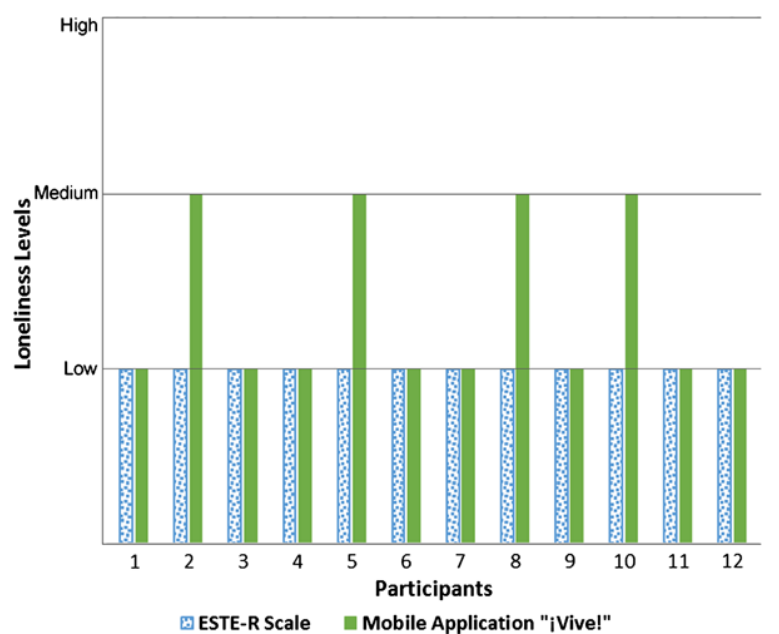

Fig. 10. Comparison results of social loneliness.

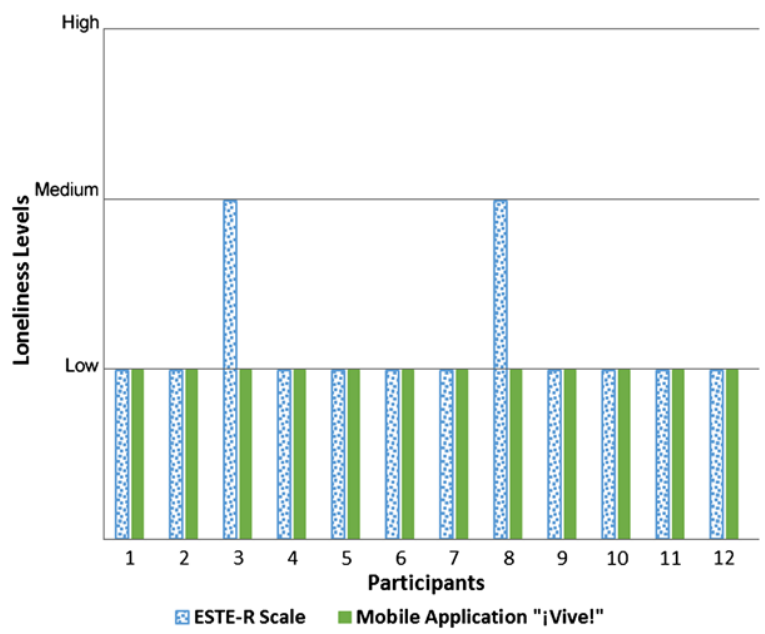

Fig. 11. Comparison results of existential crisis loneliness.

For family and spousal loneliness, outgoing and incoming family calls are relevant attributes. One possible interpretation of this finding is that older adults who keep in contact with their spouses and relatives, are at a lower risk of suffering family and spousal loneliness.

For social loneliness, friends' calls were not a relevant attribute, instead, acquaintances calls were a relevant attribute. One possible interpretation of this finding is that, despite one of the risk factors for loneliness is the death of friends, older adults who socialize with other people, have a lower risk of suffering social loneliness.

Finally, the attributes average time spent outside of the home and total of outgoings were the most impor- 
Table 12

Data collected with the application;Vive!

\begin{tabular}{lrrrrrrrrl}
\hline Num. & A & B & C & D & E & F & G & H & \multicolumn{1}{c}{ I } \\
\hline 1 & 5 & 0 & 3 & 17 & 0 & 1 & 9 & 87 & 26 \\
2 & 2 & 4 & 8 & 4 & 3 & 13 & 24 & 85.5 & 26.5 \\
3 & 2 & 0 & 0 & 2 & 0 & 4 & 9 & 88 & 24 \\
4 & 1 & 1 & 2 & 6 & 5 & 4 & 10 & 105.5 & 30.5 \\
5 & 4 & 0 & 0 & 4 & 3 & 30 & 9 & 89.5 & 22.5 \\
6 & 1 & 1 & 3 & 1 & 1 & 0 & 11 & 63 & 49 \\
7 & 11 & 1 & 12 & 2 & 1 & 9 & 10 & 54 & 58 \\
8 & 1 & 1 & 0 & 1 & 0 & 0 & 12 & 35 & 77 \\
9 & 3 & 2 & 0 & 7 & 9 & 1 & 10 & 50 & 30 \\
10 & 8 & 4 & 0 & 7 & 12 & 3 & 13 & 60 & 30 \\
11 & 1 & 0 & 0 & 31 & 12 & 2 & 15 & 50 & 40 \\
12 & 8 & 3 & 0 & 11 & 0 & 0 & 10 & 50 & 30 \\
\hline
\end{tabular}

tant attributes to infer loneliness, since these attributes were present in the four factors of loneliness. One possible interpretation of this finding is that older adults who keeps busy doing different activities like going to elderly clubs, dancing, going to the church, going to supermarket, etc., tend to be less likely to suffer from loneliness. An optimistic mind is normally a busy mind residing in a busy body and so has no little time to be unhappy [37].

\subsection{Predictive models}

In order to handle the imbalanced data, an oversampling technique (SMOTE) was applied. Overall, predictive models performance were better using the SMOTE. For only spousal loneliness, the resampling technique gave opposite results from that which was expected. This may be because civil status was the most discriminant attribute and the synthetic instances created by SMOTE caused noise and affected the classifiers algorithms performance.

In the experiment, the selected model of social loneliness produced a lower accuracy than produced by the cross-validation test, from $74 \%$ to $66.67 \%$. The type II error rate was higher than the one for the crossvalidation test, from $26 \%$ to $33 \%$. The selected models of spousal and existential crisis loneliness produced a lower accuracy than that produced by cross-validation test, but produced a type II error rate of $0 \%$. The selected model of family loneliness produced a better accuracy than that produced by cross-validation test, from $84 \%$ to $91.67 \%$, and in addition, it produced a type II error rate of $0 \%$. Based upon these results, the relevant attributes selected for social loneliness (Outgoing calls to acquaintances, Total outings, Average time outside of the home), may not have enough correlation for determining social loneliness. Nevertheless, new experiments with a larger sample are required.

\section{Conclusions}

Loneliness is considered to be one of the possible factors that causes disorders such as depression, suicide, and serious medical problems like heart illnesses [8]. An opportune diagnosis and suitable interventions, allow older adults to cope this health condition.

From psychology approach, Rubio Herrera [9] consider different aspects of the concept of loneliness: Family Loneliness, Spousal Loneliness, Social Loneliness and Existential Crisis.

In order to infer the four factors of loneliness, an evaluation of a group of activities that can be monitored by a Smartphone was carried out. From these activities, relevant attributes were identified through methods for evaluation of attributes. Using such attributes, predictive models were developed by implementing a range of classifier algorithms. Each model, went through a performance evaluations, techniques to handling imbalance data and bias. Models with better performance and lower type II error rate were implemented in a mobile application called "¡Vive!" in order to automate data collection and to infer each factor of loneliness.

In order to evaluate the selected models, an experiment using the mobile application " iVive!" was carried out. The experiment compared the results of the mo-

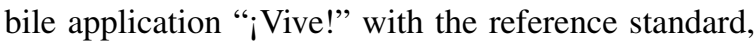
the ESTE-R scale.

Finally, the experiment results showed that the selected models have a good level of accuracy when inferring loneliness levels.

A limitation to our work is the amount of data available. It was both expensive and time-consuming to collect such data from older adults. Nevertheless, a collaborative project with geriatric institutions is currently underway, which will allow our current approach to be extended to a larger sample size, avoiding the overfitting effect.

As a future work, an implementation of a new module in the mobile application " $i V i v e ! "$ is considered. Older adults will be capable to share their loneliness levels to previous authorized caregivers and relatives, with the intention to alert them in front a risk situation. 
Another interesting future direction is to carry out a hands-on evaluation in order to assess the impact of the encouragement messages in the older adults.

\section{Acknowledgments}

We thank Ana Peña for her support in psychology issues for inferring predictive models. This research was supported by the DGEST project with Reference Num. 5049.13-P.

\section{References}

[1] E. Peña Nieto, National Development Plan. Health Sector Program 2013-2018, Government of the Mexican Republic, 2013.

[2] P. Chemor Ruíz, National Population Council, 2014, Retrieved: http://www.conapo.gob.mx/es/CONAPO/Proyecciones

[3] A. Rokach, The subjectivity of loneliness and coping with it, Psychological Reports 79(2) (1996), 475-481.

[4] D. Perlman and L.A. Peplau, Toward a social psychology of loneliness, in: Personal Relationships in Disorder, S. Duck and R. Gilmour, eds, Vol. 6, Academic Press, London, 1981, pp. 32-56.

[5] D. Barretta, D. Dantzler and W. Kayson, Factors related to loneliness, Psychological Reports 76(3) (1995), 827-830.

[6] M.A. Caldwell and L.A. Peplau, Loneliness: a cognitive analysis, Essence 2(4) (1978), 207-220.

[7] J. Tunstall, Old and Alone: A Sociological Study of Old People, Routledge and Kegan Paul, 1966, 344 pp.

[8] A. Ruiz, The frightening loneliness, 2001, Retrieved: http:// www.inteco.cl/temas/t0104a.html.

[9] R. Rubio Herrera, Loneliness in the elderly. An alternative measurement through ESTE scale, Technical report, University of Granada, Spain, 2010.

[10] D. Russell, L.A. Peplau and M.L. Ferguson, Developing a measure of loneliness, Journal of Personality Assessment 42(3) (1978), 290-294.

[11] J.C. Augusto, Ambient intelligence: the confluence of ubiquitous/pervasive computing and artificial intelligence, in: Intelligent Computing Everywhere, A. Schuster, ed., Springer, London, 2007, pp. 213-234.

[12] D.I. Tapia, A. Abraham, J.M. Corchado and R.S. Alonso, Agents and ambient intelligence: case studies, Journal of Ambient Intelligence and Humanized Computing 1(2) (2009), 8593.

[13] S. Aoki, M. Onishi, A. Kojima and K. Fukunaga, Detection of a solitude senior's irregular states based on learning and recognizing of behavioral patterns, IEEJ Transactions on Sensors and Micromachines 125(6) (2005), 259-265.

[14] Y.H. Gu, C.H. Song, S.J. Yoo, D.I. Han, J.H. Choi and S.J. Park, Recognition of loneliness of the elderly people in ubiquitous computing environment, Advances in Computational Science and Engineering 28 (2009), 60-72.

[15] J. Petersen, D. Austin, J.A. Kaye, M. Pavel and T.L. Hayes, Unobtrusive in-home detection of time spent out-of-home with applications to loneliness and physical activity, IEEE Journal of Biomedical and Health Informatics 18(5) (2014), 1590-6.

[16] Ç. Tan, M. Pamuk and A. Dönder, Loneliness and mobile phone, Procedia - Social and Behavioral Sciences 103 (2013), 606-611.

[17] M. Hall, E. Frank, G. Holmes, B. Pfahringer, P. Reutemann and I.H. Witten, The WEKA data mining software: an update, SIGKDD Explorations 11(1) (2009).

[18] I.H. Witten, E. Frank and M. Hall, Data Mining: Practical Machine Learning Tools and Techniques, 3rd edn, 2011, 630 pp.

[19] D.S. Moore, Basic Applied Statistics, 2nd edn, Antoni Bosch editor, 2005.

[20] M.A. Hall, Correlation-based feature selection for machine learning, The University of Waikato, 1999.

[21] R. Kohavi and G.H. John, Wrappers for feature subset selection, Artificial Intelligence 97(1-2) (1997), 273-324.

[22] X. Wu, V. Kumar, J.R. Quinlan, J. Ghosh, Q. Yang, H. Motoda, G.J. McLachlan, A. Ng, B. Liu, P.S. Yu, Z.-H. Zhou, M. Steinbach, D.J. Hand and D. Steinberg, Top 10 algorithms in data mining, Knowledge and Information Systems 14(1) (2007), 137.

[23] G.H. John and P. Langley, Estimating continuous distributions in Bayesian classifiers, in: UAI'95 Proc. of the Eleventh Conference on Uncertainty in Artificial Intelligence, 1995, pp. 338-345.

[24] N. Landwehr, M. Hall and E. Frank, Logistic model trees, Machine Learning 59(1-2) (2005), 161-205.

[25] J.C. Platt, Fast training of support vector machines using sequential minimal optimization, in: Advances in Kernel Methods: Support Vector Learning, B. Schölkopf, C.J.C. Burges and A.J. Smola, eds, MIT Press, 1999, pp. 185-208.

[26] D.W. Aha, D. Kibler and M.K. Albert, Instance-based learning algorithms, Machine Learning 6(1) (1991), 37-66.

[27] Y. Freund and R.E. Schapire, Experiments with a new boosting algorithm, in: Thirteenth International Conference on Machine Learning, 1996, pp. 148-156.

[28] R.C. Holte, Very simple classification rules perform well on most commonly used datasets, Machine Learning 11(1) (1993), 63-90.

[29] R. Quinlan, C4.5: Programs for Machine Learning, Morgan Kaufmann Publishers Inc., 1993.

[30] L. Breiman, J.H. Friedman, R. Olshen and C.J. Stone, Classification and Regression Trees, Wadsworth International Group, 1984, 368 pp.

[31] N.V. Chawla, K.W. Bowyer, L.O. Hall and W.P. Kegelmeyer, SMOTE: synthetic minority over-sampling technique, Journal of Artificial Intelligence Research 16 (2002), 321-357.

[32] S. Banoo, D. Bell, P. Bossuyt, A. Herring, D. Mabey, F. Poole, P.G. Smith, N. Sriram, C. Wongsrichanalai, R. Linke, R. O'Brien, M. Perkins, J. Cunningham, P. Matsoso, C.M. Nathanson, P. Olliaro, R.W. Peeling and A. Ramsay, Evaluation of diagnostic tests for infectious diseases: general principles, Nature Reviews Microbiology 8 (2010), 17-29.

[33] C. Manterola, How to read an article about diagnostics tests, Clinic Medical Journal Las Condes 20(5) (2009), 708-717.

[34] R. Riegelman, Studying a Study and Testing a Test: How to Read the Medical Evidence, Lippincott Williams \& Wilkins, 2005.

[35] R.H. Fletcher, S.W. Fletcher and G.S. Fletcher, Clinical Epidemiology: The Essentials, Lippincott Williams \& Wilkins, 2012. 
[36] R.R. Herrera, Loneliness in Spanish elderly, Madrid, Spain, 2004, pp. 1-21, Technical report, Inmerso, Retrieved: http://www.imsersomayores.csic.es/documentos/documentos/ rubio-soledad-01.pdf.
[37] R.A. Punjabi, Love-Life of a Smile, 1st edn, AuthorHouse, 2009. 